\title{
Giant outburst of EXO 2030+375: pulse-phase resolved analysis of INTEGRAL data
}

\author{
D. Klochkov ${ }^{1}$, A. Santangelo ${ }^{1}$, R. Staubert ${ }^{1}$, and C. Ferrigno ${ }^{2}$ \\ 1 Institut für Astronomie und Astrophysik, Universität Tübingen (IAAT), Sand 1, 72076 Tübingen, Germany \\ e-mail: klochkov@astro.uni-tuebingen.de \\ 2 INAF IFC-Pa, via U. La Malfa 153, 90146 Palermo, Italy
}

Received 25 July 2008 / Accepted 6 September 2008

\begin{abstract}
In June-September 2006 the Be/X-ray binary EXO 2030+375 experienced the second giant outburst since its discovery. The source was shown to have a complicated pulse-averaged X-ray spectral continuum with possible evidence of cyclotron absorption features. In this paper we present the first pulse-phase resolved analysis of the broad band X-ray spectra of EXO 2030+375 obtained with the INTEGRAL observatory close to the maximum and during the decay phase of the giant outburst. We report a strong variability of the spectrum with pulse phase. Alternative spectral continuum models are discussed. The dependence of the spectral parameters on pulse phase during the maximum of the outburst and the evolution of the pulse profiles with time are qualitatively consistent with the pulsar's emission diagram changing from the fan-beam geometry close to the maximum of the outburst to a combination of pencil and fan beams (of comparable intesities) at the end of the decay phase. Evidence of a cyclotron absorption line around $63 \mathrm{keV}$ at the pulse phase interval preceeding the main peak of the pulse profile is present in the spectrum obtained close to the maximum of the outburst.
\end{abstract}

Key words. stars: neutron - X-rays: binaries - accretion, accretion disks

\section{Introduction}

The transient accreting pulsar EXO 2030+375 belongs to the most common type of X-ray pulsar systems - the Be/X-ray binaries. Such systems form a subclass of high mass X-ray binaries. They consist of a pulsar and a $\mathrm{Be}$ (or Oe) companion, a main-sequence star of spectral type B (or O) that shows Balmer emission lines (see e.g. Slettebak 1988, for a review). The line emission is believed to be associated with an equatorial outflow of material expelled from the rapidly rotating Be star that probably forms a quasi-Keplerian disk around its equator (Hanuschik 1996; Quirrenbach et al. 1997). If the disk reaches a radius comparable to the periastron separation, then disk gas accreted by the neutron star can power a significant (and usually transient) $\mathrm{X}$-ray source. havior:

Be/X-ray binaries typically show two types of outburst be-

1. normal (or type I) outbursts. They are characterized by relatively low X-ray luminosities $L_{\mathrm{X}} \sim 10^{36}-10^{37} \mathrm{erg} \mathrm{s}^{-1}$, low spin-up rates (if any), and recurrence at the orbital period (or its multiples). Such outbursts last from days to weeks and are associated with the periastron passages of the neutron star;

2. giant (or type II) outbursts. They are characterized by higher $\mathrm{X}$-ray luminosities $L_{\mathrm{X}} \gtrsim 10^{37} \mathrm{erg} \mathrm{s}^{-1}$ and high spin-up rates. Such outbursts occur irregularly. They last several weeks and are not correlated with any particular orbital phase. The typical time between outbursts is around several years. Giant outbursts are thought to stem from a dramatic expansion of the disk surrounding the Be star, leading to the formation of an accretion disk around the compact object.

EXO $2030+375$ is one of the best-studied Be/X-ray binaries. It was discovered with the EXOSAT satellite during a giant outburst in 1985 (Parmar et al. 1989b). The optical companion of the pulsar is a B0 Ve star identified by optical and infrared observations of the EXOSAT error circle (Motch \& Janot-Pacheco 1987; Janot-Pacheco et al. 1988; Coe et al. 1988). The orbital period and eccentricity of the system are $\sim 46 \mathrm{~d}$ and $\sim 0.42$, respectively (Wilson et al. 2002). The period of X-ray pulsations is $\sim 42 \mathrm{~s}$. There were two giant outbursts in the history of observations of EXO 2030+375. During the first one in 1985 (when the pulsar was discovered), the X-ray luminosity of the source reached a value of $L_{1-20 \mathrm{keV}} \sim 2 \times 10^{38} \mathrm{erg} \mathrm{s}^{-1}$ (assuming a distance of $7.1 \mathrm{kpc}$, Wilson et al. 2002). The spin frequency of the pulsar changed dramatically, with a spin-up timescale $-P / \dot{P} \sim 30 \mathrm{yr}$ indicating the formation of an accretion disk around the neutron star. The second giant outburst took place in June-September 2006 (Corbet \& Levine 2006; Klochkov et al. 2007) and was again accompanied by a strong spin-up of the neutron star. The X-ray luminosity at the maximum of the outburst was slightly lower than during the 1985 giant outburst: $L_{1-20 \mathrm{keV}} \sim 1.2 \times 10^{38} \mathrm{erg} \mathrm{s}^{-1}$ (Wilson \& Finger 2006).

During the 2006 giant outburst the source was observed several times with the INTEGRAL satellite. A preliminary pulse-averaged spectral analysis of some of these observations are presented in Klochkov et al. (2007). A detailed analysis of pulse-averaged $R X T E$ spectra obtained during the outburst was performed by Wilson et al. (2008). It has been shown that the pulse averaged X-ray continuum of the source has a complicated shape and cannot be modeled by a simple power law/cutoff model. Wilson et al. (2008) include an absorption line at $\sim 10 \mathrm{keV}$ (which they interpreted as a cyclotron line) in their spectral model, whereas Klochkov et al. (2007) have shown that the spectrum can be fitted equally well without the absorption line, but including a broad emission "bump" at $\sim 15 \mathrm{keV}$. 


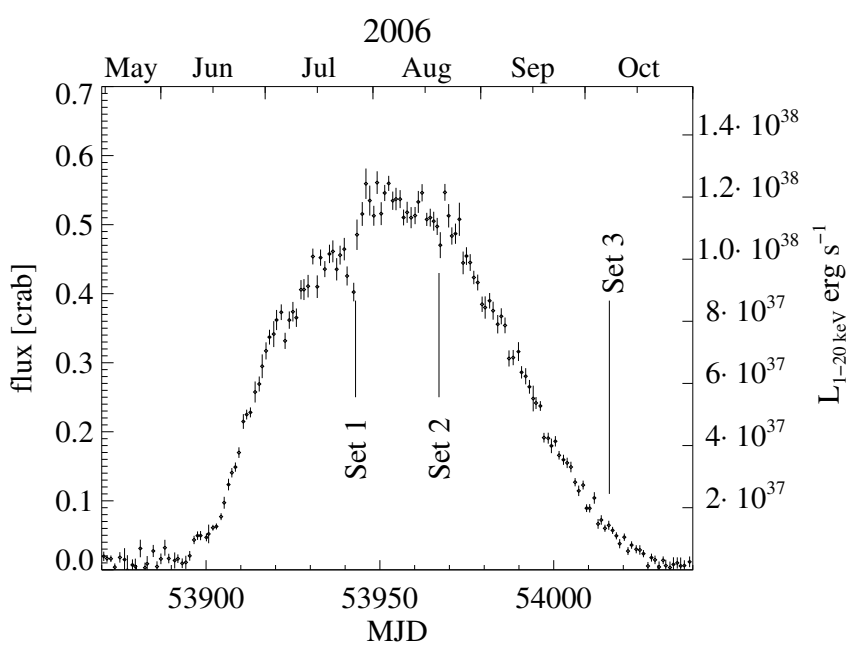

Fig. 1. The RXTE/ASM light curve of EXO 2030+375 showing the 2006 giant outburst. Times of INTEGRAL observations analyzed in this work are marked with vertical lines. The observations are referred to as Set 1 , Set 2 , and Set 3 in the text.

Here we present for the first time pulse-phase resolved broad band (3-150 keV) spectra of EXO 2030+375 during a giant outburst. For our analysis we used all the available INTEGRAL data taken during the 2006 outburst. The X-ray continuum of the source shows strong variability with pulse phase, with some features present only at particular pulse-phase intervals. The description of observations that we used is provided in Sect. 2. Details of data processing are described in Sect. 3. Sections 4 and 5 are devoted to the analysis of the data close to the maximum and during the decay of the outburst, respectively. The results are discussed in Sect. 6 and briefly summarized in Sect. 7.

\section{Observations}

The INTErnational Gamma Ray Astrophysics Laboratory (INTEGRAL, Winkler et al. 2003) performed three pointed observations of EXO 2030+375 during its giant outburst in JuneSeptember 2006. Two observations (on 19-20 August and 2325 September) were done close to the maximum of the outburst. The third one (on 6-8 October) was performed at the end of the decay phase, when the X-ray luminosity dropped by a factor of $\sim 10$ with respect to the maximum of the outburst. A part of the RXTE/ASM light curve ${ }^{1}$ including the giant outburst is shown in Fig. 1. The times of the INTEGRAL observations are indicated and referred to as Set 1, Set 2, and Set 3 throughout the paper.

Observations corresponding to Sets 1 and 2 were done when the source was at similar luminosity levels $\left(L_{1-20} \mathrm{keV} \sim\right.$ $10^{38} \mathrm{erg} \mathrm{s}^{-1}$ ) before and after the maximum of the outburst. A preliminary analysis of the two data sets was presented by Klochkov et al. (2007) who showed that spectral and timing characteristics of the source during the two observations were similar. Due to the high X-ray flux, the data allowed us to perform a detailed pulse-phase resolved spectral analysis of EXO 2030+375 using twelve phase bins. During the observations corresponding to Set 3, the X-ray luminosity of the source was at a level typical of normal (Type I) outbursts by this sys-

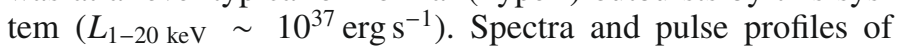
the source during these observations were considerably different with respect to those in Sets 1 and 2. In spite of a poorer

\footnotetext{
${ }^{1}$ We used the results provided by the ASM/RXTE team.
}

Table 1. Summary of observations.

\begin{tabular}{cccc}
\hline \hline Obs. & $\begin{array}{c}\text { Obs.time } \\
(\mathrm{ks})\end{array}$ & $\begin{array}{c}\text { Mean } \\
\text { MJD }\end{array}$ & $\begin{array}{c}\text { Mean ASM } \\
\text { flux (mCrab) }\end{array}$ \\
\hline Set 1 & 62 & 53942.9 & 450 \\
Set 2 & 140 & 53967.6 & 500 \\
Set 3 & 122 & 54015.2 & 60 \\
\hline
\end{tabular}

statistics, the data taken in Set 3 still allowed us to perform pulsephase resolved spectral analysis, although with a coarser binning (four bins) in pulse phase. Table 1 contains the summary of the observations analyzed in this work.

\section{Data processing}

For our analysis we used the data obtained with the instruments IBIS/ISGRI (20-300 keV, Ubertini et al. 2003) and JEM-X (3$30 \mathrm{keV}$, Lund et al. 2003) onboard INTEGRAL. To perform the standard data reduction, the Off-line Science Analysis (OSA) software (version 6) was used (Courvoisier et al. 2003). To construct energy-resolved pulse profiles we used the software developed at IASF, Palermo (Mineo et al. 2006). While extracting spectra and pulse profiles of EXO $2030+375$, two other bright sources in the field of view of the INTEGRAL instruments, Cyg X-1 and Cyg X-3, were included to the extraction catalog used by the analysis software, which removes the contamination of EXO 2030+375. All other sources in the field of view are much weaker than EXO 2030+375 and therefore do not provide any noticeable contamination. The spectral analysis of the observations was performed using the XSPEC v.11.3.21 spectral fitting package (Arnaud 1996). Following the OSA User Manuals ${ }^{2}$, we added a systematic error to the final count rates at a level of $1 \%$ for ISGRI and $2 \%$ for $J E M-X$ to account for small-scale uncertainties in the response matrices of the respective instruments.

To extract X-ray pulse profiles and to define pulse phase intervals for pulse phase resolved spectra, all photon arrival times were translated into the solar system barycenter and corrected for orbital motion in the binary. The orbital parameters were taken from Wilson et al. (2005): $P_{\text {orb }}=46.0202(2) \mathrm{d}$, $T_{\text {peri }}=\operatorname{MJD} 51$ 099.43(2), $a \sin i=238(2)$ lt-sec, $e=0.416(1)$, $\omega=210.8$ (4). The pulse periods and associated derivatives were determined for each of the three observational sets individually by employing initial epoch-folding and a subsequent phaseconnection analysis similar to Ferrigno et al. (2007) and Deeter et al. (1981). To perform phase-connection, we used a patternmatching technique applied to well-defined pulse profiles from a sufficient number of pulses. Any variation in the pulse shape inside each observation is marginal and does not affect our method. The determined periods and period derivatives are summarized in Table 2. Values corresponding to Sets 1 and 2 are slightly improved with respect to those reported in Klochkov et al. (2007).

\section{Maximum of the outburst}

Observations corresponding to Sets 1 and 2 were performed close to the maximum of the outburst (Fig. 1). During Set 1, which is roughly half as long as Set 2 (see Table 1), the main target of observations was Cyg X-3 located $4^{\circ}$ away from EXO 2030+375. Therefore, the statistics of the EXO 2030+375 data obtained during Set 1 is noticeably worse than during Set 2.

\footnotetext{
${ }^{2}$ http://isdc.unige.ch/index.cgi?Support+documents
} 

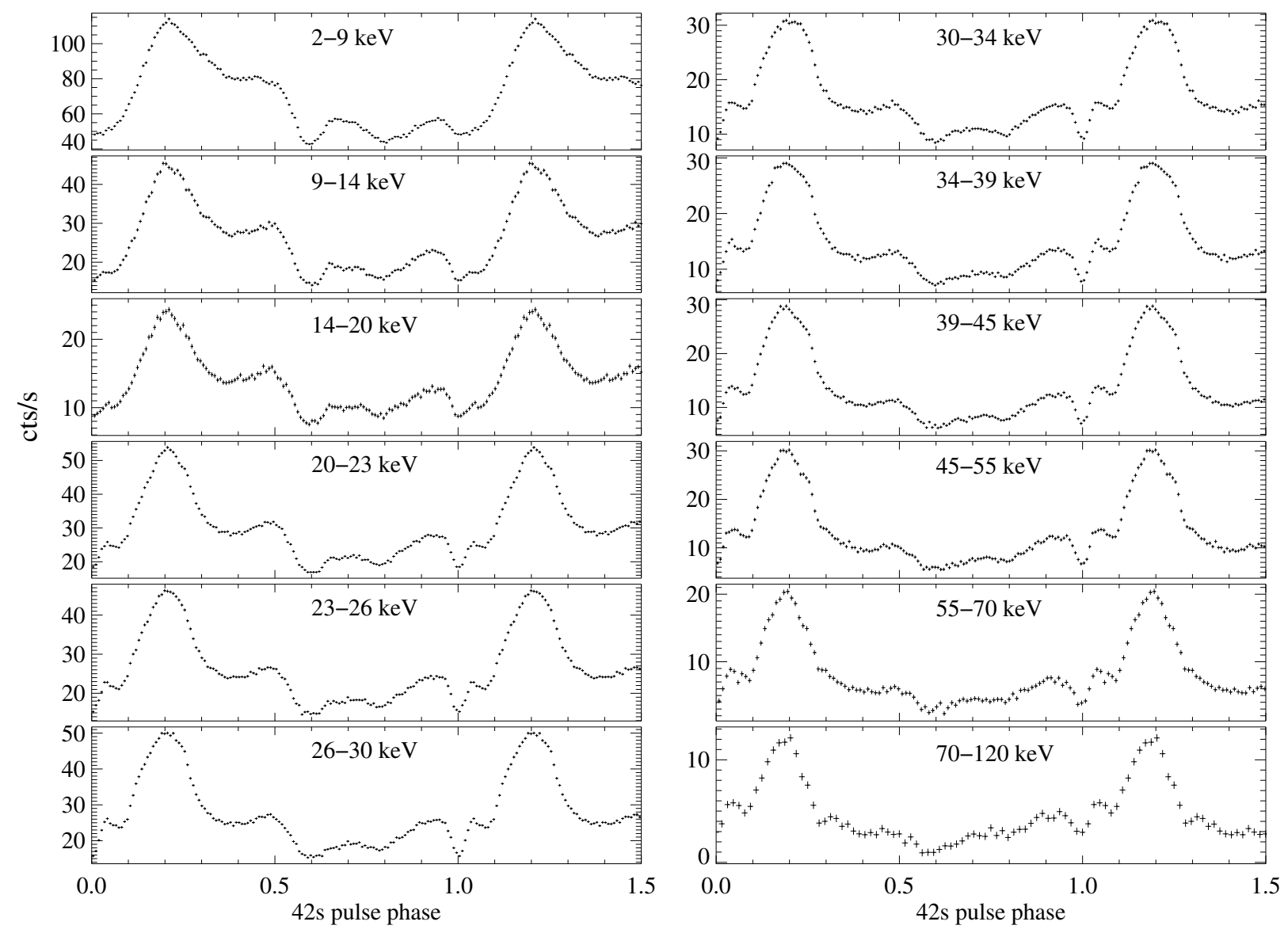

Fig. 2. Energy-resolved pulse profiles of EXO 2030+375 obtained with JEM-X (below $20 \mathrm{keV}$ ) and IBIS/ISGRI (above $20 \mathrm{keV}$ ) in Set 2 (where the data have the best statistics).

Table 2. The pulse periods and associated derivatives for three sets of observations analyzed in this work.

\begin{tabular}{cccc}
\hline \hline Observation & $\begin{array}{c}\text { Reference } \\
\text { epoch (MJD) }\end{array}$ & $\begin{array}{c}P \\
{[\mathrm{~s}]}\end{array}$ & $\begin{array}{c}-\mathrm{d} P / \mathrm{d} t \\
{\left[10^{-8} \mathrm{~s} / \mathrm{s}\right]}\end{array}$ \\
\hline & & & \\
Set 1 & 53942.240864 & $41.58084(3)$ & $2.96(21)$ \\
Set 2 & 53966.392169 & $41.519270(5)$ & $2.758(20)$ \\
Set 3 & 54014.010200 & $41.45425(6)$ & $0.22(23)$ \\
\hline
\end{tabular}

Note. Uncertainties in parentheses $(68 \%)$ refer to the last digit(s).

As a result, our pulse-phase resolved analysis of the maximum of the outburst is mainly driven by the data taken in Set 2 .

To explore spectral changes with pulse phase, we constructed pulse-phase resolved spectra and energy-resolved pulse profiles. While the former allow detailed study of spectral parameters as a function of pulse phase, the latter can be used to explore the intensity of different pulse-profile components (peaks, dips, etc.) in different energy ranges without relying on any particular spectral function.

\subsection{Energy-resolved pulse profiles}

Figure 2 presents energy-resolved background-subtracted pulse profiles of the source obtained during the observations corresponding to Set 2 . The corresponding pulse period and its derivative, as well as the zero epoch, are provided in Table 2 . The pulse profiles in the energy bands below $20 \mathrm{keV}$ were obtained from the JEM-X data, while the IBIS/ISGRI data were used for energies above $20 \mathrm{keV}$. Several components can be distinguished in the profiles: the main peak at phase $\sim 0.2$ followed by a fainter component at phase $\sim 0.5$ (which we will refer to as the "trailing shoulder") and two smaller peaks in the interpulse interval 0.6-1.0. The sharpest detail of the profile is a narrow dip preceding the main peak. Phase 0.0 was arbitrarily chosen to coincide with the dip in the average ISGRI pulse profile. As one can see, the shape of the profile changes smoothly with energy indicating variations in the $\mathrm{X}$-ray spectrum with pulse phase. The pulse fraction of the source determined as $\left(F_{\max }-F_{\min }\right) /\left(F_{\max }+F_{\min }\right)$ (where $F_{\max }$ and $F_{\min }$ are fluxes in the maximum and minimum of the pulse profile, respectively) as a function of energy is shown in Fig. 3. One can see a bump-like structure around $\sim 10$ $20 \mathrm{keV}$ that is probably related to the continuum feature in this energy range (the "bump" or the absorption line, see Sects. 1 and 4.2). Above $\sim 20 \mathrm{keV}$, the pulse fraction seeply increases.

\subsection{Pulse-resolved spectra}

As one can conclude from the dependence of the pulse profile shape on energy, the X-ray spectrum of EXO 2030+375 clearly varies with pulse phase. We have therefore performed a separate analysis of the spectra accumulated in different pulse phase intervals. Phase binning was chosen to provide similar statistics of spectra in each bin and to have better phase resolution around the main peak where the most rapid spectral changes are expected. The binning was chosen a priori based solely on the shape of the pulse profile. No further adjustments were made after the 


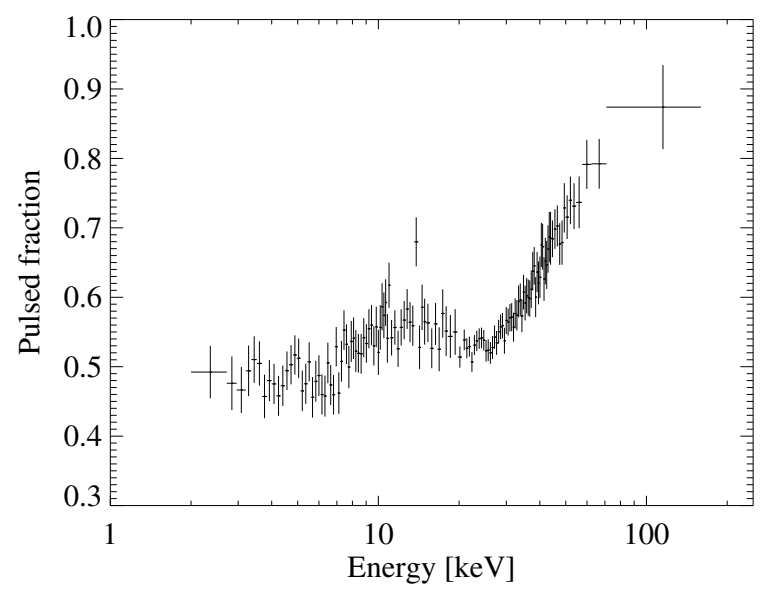

Fig. 3. The pulse fraction of EXO $2030+375$ as a function of energy.

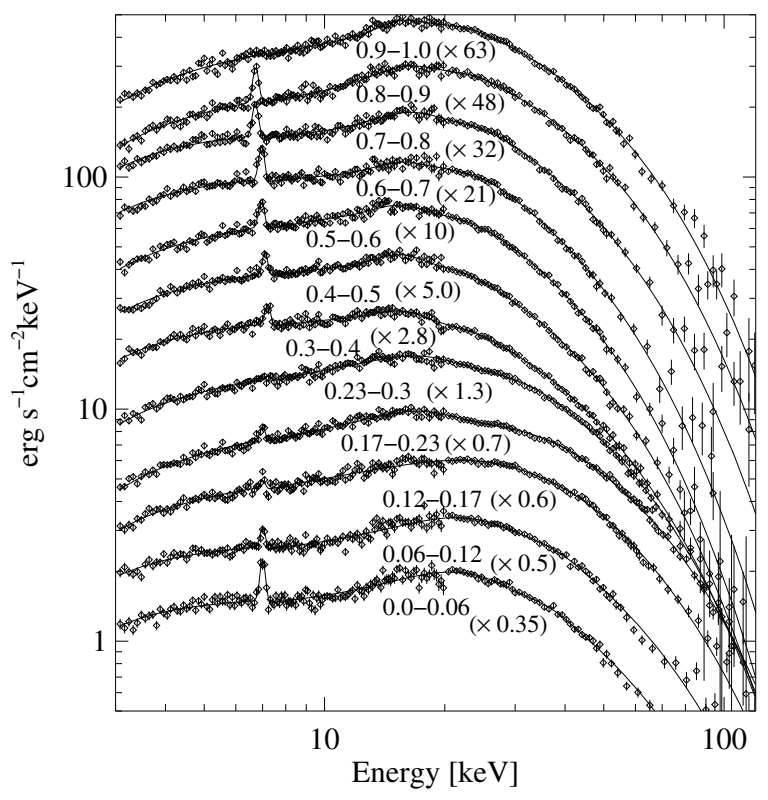

Fig. 4. Pulse-resolved broad band X-ray spectra of EXO 2030+375 obtained in Set 2. Phase bins are indicated (phase zero is the same as in Fig. 2). Values in parenthesis indicate the multiplicative factor applied to the flux in each spectrum to avoid overlaps. The solid line represents the fit of the spectra using Model I (see text for the model details).

spectra had been obtained. Figure 4 shows unfolded pulse phaseresolved spectra of the source (shifted vertically with respect to each other to avoid overlaps). The pulse phase is defined in the same way as in the previous section. Variability of the spectral continuum is clearly seen.

As pointed out in Sect. 1, the pulse-averaged spectral continuum of the source is rather complicated and cannot be modeled by any of the simple spectral functions (a power law modified at higher energies by an exponential cutoff), which are usually used to fit spectra of accreting pulsars. In an attempt to model the spectrum Wilson et al. (2008) modified a power law/cutoff model by a Gaussian absorption line at $\sim 10 \mathrm{keV}$, while Klochkov et al. (2007) included a broad Gaussian emission component at $\sim 15 \mathrm{keV}$ instead. In this work we tried to use both models to fit the pulse-resolved spectra. The reduced $\chi^{2}$ in all phase bins is very similar for the two models. This does not allow one to ultimately choose between the two spectral functions. The two models are referred to as Model I (with a "bump") and Model II (with an absorption line at $10 \mathrm{keV}$ ) throughout the paper.
Below we describe the spectral functions corresponding to the two models. The common part of Models I and II is the power law/cutoff continuum smoothed at the cutoff energy by a thirdorder polynomial:

$I_{\text {cont }}=K \cdot \begin{cases}E^{-\Gamma}, & \text { if } E \leq E_{\text {cutoff }}-\Delta E \\ E^{-\Gamma} \cdot \exp \left(-\frac{E-E_{\text {cutoff }}}{E_{\text {fold }}}\right), & \text { if } E>E_{\text {cutoff }}+\Delta E \\ A E^{3}+B E^{2}+C E+D, & \text { if } E_{\text {cutoff }}-\Delta E<E< \\ & E_{\text {cutoff }}+\Delta E,\end{cases}$

where $K$ is the normalization coefficient, $E$ the photon energy; $\Gamma, E_{\text {cutoff }}, E_{\text {fold }}$, and $\Delta E$ are model parameters. Numerical coefficients $A, B, C$, and $D$ are chosen to obey the condition of continuity for the function and its first derivative at the points $\left(E_{\text {cutoff }}-\Delta E\right)$ and $\left(E_{\text {cutoff }}+\Delta E\right)$. Model I includes a broad Gaussian emission component (a "bump") around $15 \mathrm{keV}$ :

$I_{\text {Model I }}=I_{\text {cont }}+K_{\text {bump }} \exp \left\{-\frac{\left(E-E_{\text {bump }}\right)^{2}}{2 \sigma_{\text {bump }}^{2}}\right\}$,

where $E_{\text {bump }}$ and $\sigma_{\text {bump }}$ are the energy and width of the "bump", and $K_{\text {bump }}$ is the numerical constant describing the intensity of the component. In Model II, the power law/cutoff continuum $I_{\text {cont }}$ is modified by a multiplicative absorption line with a Gaussian optical depth profile:

$I_{\text {Model II }}=I_{\text {cont }} \cdot \exp \left\{-\tau_{\text {line }} \exp \left(-\frac{\left(E-E_{\text {line }}\right)^{2}}{2 \sigma_{\text {line }}^{2}}\right)\right\}$,

where $E_{\text {line }}, \sigma_{\text {line }}$, and $\tau_{\text {line }}$ are the centroid energy, width, and the central depth of the line, respectively. Additionally, we multiplied the functions $I_{\text {Model I }}$ and $I_{\text {Model II }}$ by the factor $\exp \left[-N_{\mathrm{H}} \sigma_{\text {bf }}(E)\right]$ describing the low-energy absorption by cold matter in the line of sight. Here, $\sigma_{\mathrm{bf}}(E)$ is the photoabsorption cross-section per hydrogen atom for matter of cosmic abundances (Bałucińska-Church \& McCammon 1992) used in the phabs model of XSPEC, and $N_{\mathrm{H}}$ is the equivalent hydrogen column density. We also added a Gaussian emission line to model the iron fluorescence line at $\sim 6.4 \mathrm{keV}$. The latter, however, improves the fit only slightly. For all pulse-phase resolved spectra the value of $N_{\mathrm{H}}$ was fixed to $2 \times 10^{22} \mathrm{~cm}^{-2}$ (the average of the best-fit values found in all phase bins). It was also found that the energy of the "bump" in Model I does not change significantly with pulse phase being close to $15 \mathrm{keV}$. It was, therefore, fixed to this value for all phase bins.

Figures 5 and 6 show the most important spectral parameters obtained using the two models as a function of pulse phase. The data correspond to the observations performed in Set 2, which have the best statistics. Vertical error bars correspond to $90 \%$-uncertainties. Note that the pulse phase range shown in the figures is from 0.0 to 1.5 so that some data points are repeated (the same is true for Fig. 10).

The same kind of analysis was performed using the observations corresponding to Set 1 (slightly before the maximum of the outburst). Due to poorer statistics, we had to use a coarser binning compared to Set 2 . The spectral parameters of the pulseresolved spectra were less constrained in this case. However, the behavior of the parameters agrees with what is found in Set 2. We also notice that the best-fit parameters of the pulse-averaged spectra taken in Sets 1 and 2 are also consistent with each other (see Table 3 in Klochkov et al. 2007).

Residuals left after fitting the spectrum by Models I and II from phase bin $0.06-0.12$ obtained in Set 2 reveal an absorption feature at $\sim 63 \mathrm{keV}$ (see Fig. 7). Inclusion of a Gaussian absorption line at this energy flattens the residuals. The best-fit energy of the line is $63.6_{-2.9}^{+3.7} \mathrm{keV}$ for Model I and $63.3_{-2.9}^{+4.0} \mathrm{keV}$ for 


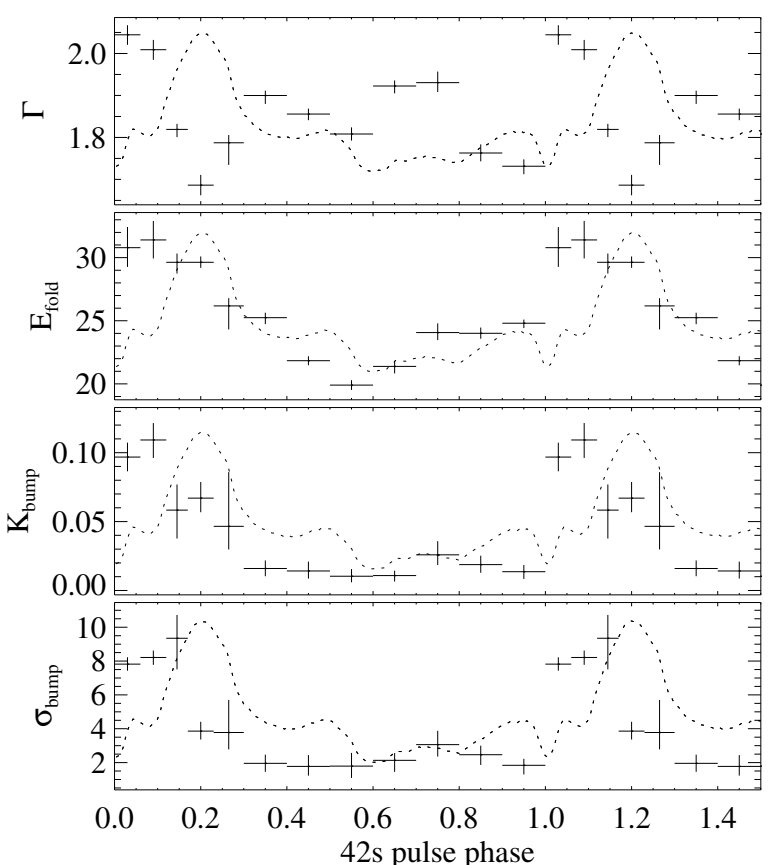

Fig. 5. Best-fit parameters of Model I (see text) as a function of pulse phase obtained by fitting the spectra from Set 2. The dotted curve shows the averaged ISGRI $(20-120 \mathrm{keV})$ pulse profile scaled and shifted vertically to match the plot. Vertical error bars correspond to 90\%-uncertainties.

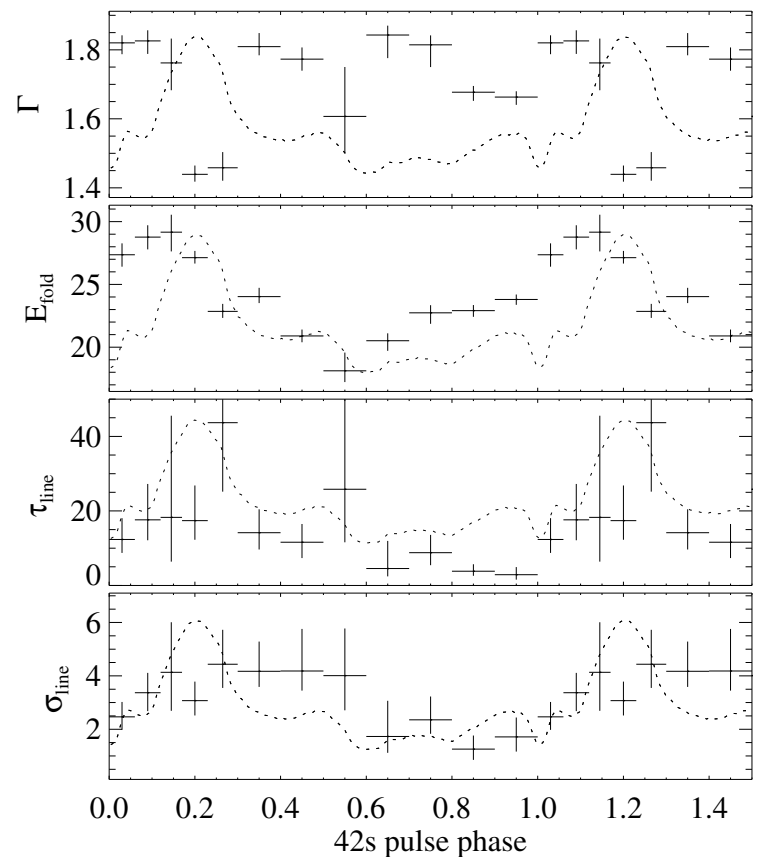

Fig. 6. Best-fit parameters of Model II (see text) as a function of pulse phase obtained by fitting the spectra from Set 2. As in Fig. 5, the dotted curve shows the ISGRI pulse profile. Vertical error bars correspond to $90 \%$-uncertainties.

Model II. We checked the presence and the energy of the feature using other possible continuum models, such as Fermi-Dirac cutoff (Tanaka 1986) and the so-called negative and positive power-law times EXponential model (NPEX, Makishima et al. 1999), including a "bump" or an absorption line at $10 \mathrm{keV}$ to match the continuum. It was found that the presence and the energy of the feature are independent of the choice of the spectral

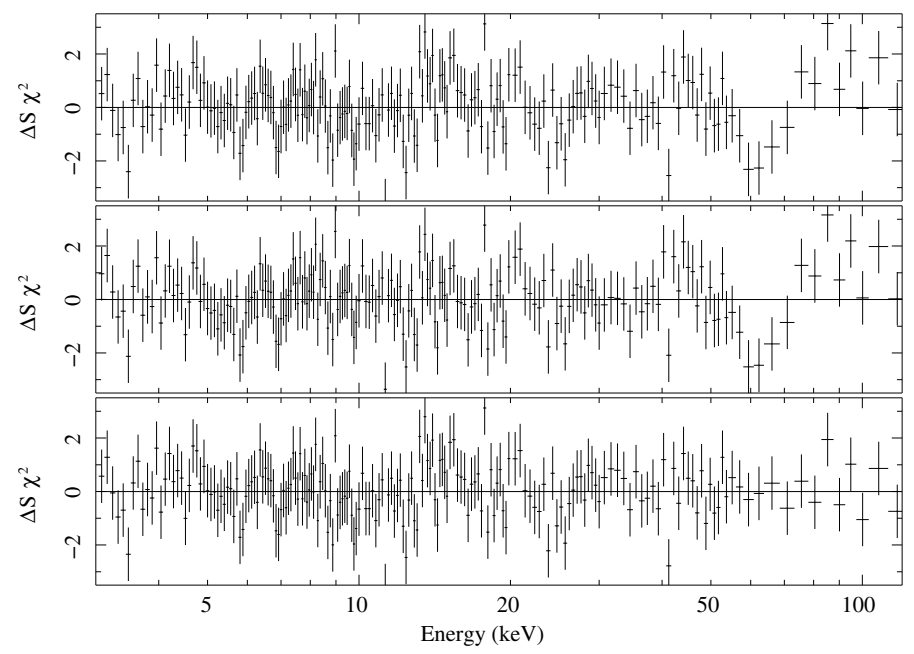

Fig. 7. Residuals of the spectrum from the phase bin $0.06-0.12$ fitted with Model I (top) and Model II (middle). The bottom panel shows the residuals after fitting the spectrum with Model I where a Gaussian absorption line at $\sim 63.6 \mathrm{keV}$ is included (the corresponding fit with Model II looks very similar).

function. The F-test probability that the line is due to statistical fluctuations is $\sim 3 \times 10^{-5}$ (see however Protassov et al. 2002, about non-applicability of the F-test to line-like features).

\section{Decay of the outburst}

\subsection{Energy-resolved pulse profiles}

As mentioned in Sect. 2, during Set 3, INTEGRAL caught the source at the end of the outburst's decay phase when the X-ray luminosity was $\sim 10$ times lower than the maximum of the outburst (Fig. 1). Due to lower statistics we used much coarser binning in energy (for pulse profiles) and pulse phase (for pulsephase resolved spectra) than in Set 2 (4 instead of 12 in both cases). The resulting pulse profiles are shown in Fig. 8. As before, the corresponding pulse period, its derivative, and the epoch zero can be found in Table 2 . To choose the time of phase zero in accordance with the one used for Set 2 we used the dependence of the pulse profiles shape on the X-ray luminosity presented in Fig. 1 of Parmar et al. (1989a). The dependence allows one to identify features in the profiles (e.g. the dip around pulse phase 0.6 ) in both observations (Sets 1 and 2) and choose the zero epoch for both observations consistently.

The dependence of the pulse profile on energy in Set 3 (Fig. 8) is mainly characterized by a decreasing relative amplitude of the peak at phase $\sim 0.9$ with respect to the main peak (phase $\sim 0.2$ ) with energy. The sharp dip seen in the $2-10 \mathrm{keV}$ at phase $\sim 0.6$ almost disappears at higher energies. Generally, one can see that the shape of the pulse profiles is substantially different as compared to the profiles obtained close to the maximum of the outburst at similar energies. In Sect. 6 we show that the changes could be qualitatively explained if assuming that a pencil beam component appears at the end of the outburst.

\subsection{Pulse-phase resolved spectra}

To produce pulse-phase resolved spectra from the data obtained in Set 3, we used four phase bins. They cover each of the two peaks and two phase intervals of the interpulse. The spectral continuum can be modeled well by the power law/cutoff function 


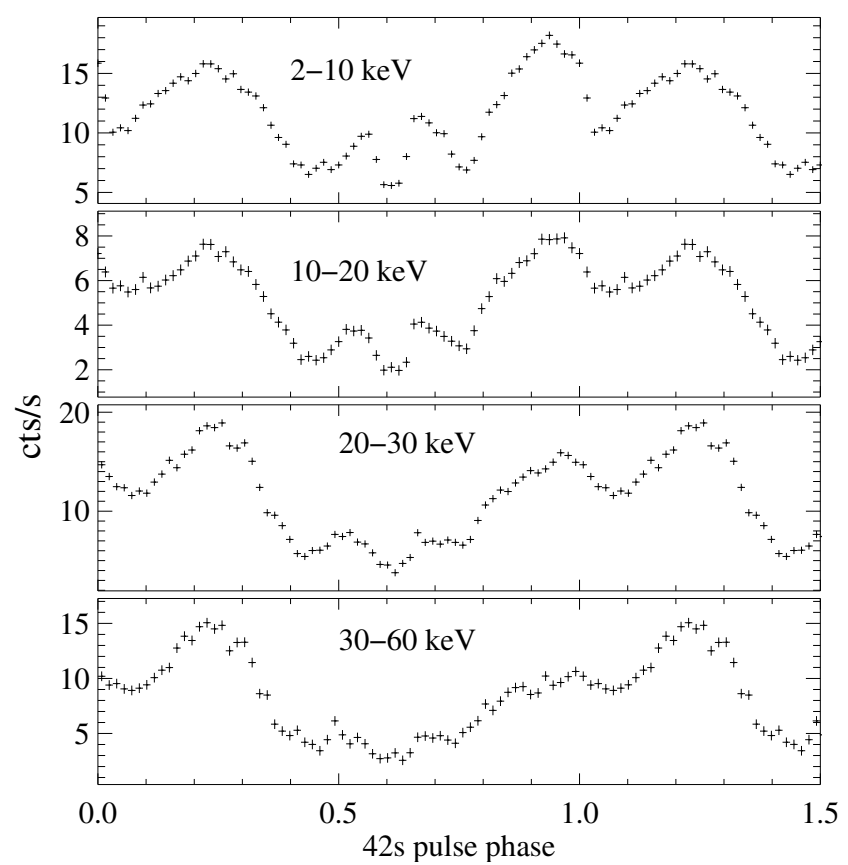

Fig. 8. Energy-resolved pulse profiles of EXO 2030+375 obtained with $J E M-X$ (below $20 \mathrm{keV}$ ) and IBIS/ISGRI (above $20 \mathrm{keV}$ ) in Set 3 (at the end of the outburst).

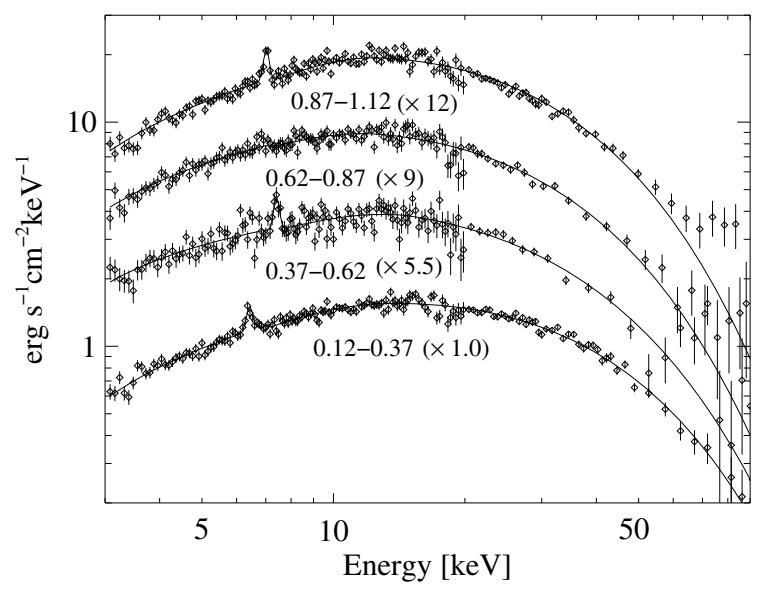

Fig. 9. Pulse-phase resolved broad band X-ray spectra of EXO 2030+375 obtained in Set 3. Phase bins are indicated (phase zero is the same as in Figs. 8). Values in parentheses indicate the multiplicative factor applied to the flux in each spectrum to avoid overlaps. The solid line represents the fit of the spectra using the power law/cutoff model (see text for the model details).

provided by Eq. (1) without inclusion of the "bump" or the $10 \mathrm{keV}$ absorption line. Like in Sect. 4.2, we modified the model at lower energies by photoabsorption (with $N_{\mathrm{H}} \sim 2 \times 10^{22} \mathrm{~cm}^{-2}$ ) and added a Gaussian line to model the iron fluorescence emission at $\sim 6.4 \mathrm{keV}$. Figure 9 represents the unfolded pulse-phase resolved spectra fitted with the described model and shifted vertically with respect to each other in order to avoid overlaps. (This was done multiplying the flux in each spectrum by a numerical factor indicated in parentheses.) Significant variation in the continuum is clearly seen. The pulse phase is defined in the same way as in Fig. 8.

Figure 10 shows the photon index $\Gamma$ and the folding energy $E_{\text {fold }}$ as a function of pulse phase during Set 3. Vertical error bars correspond to $90 \%$-uncertainties.

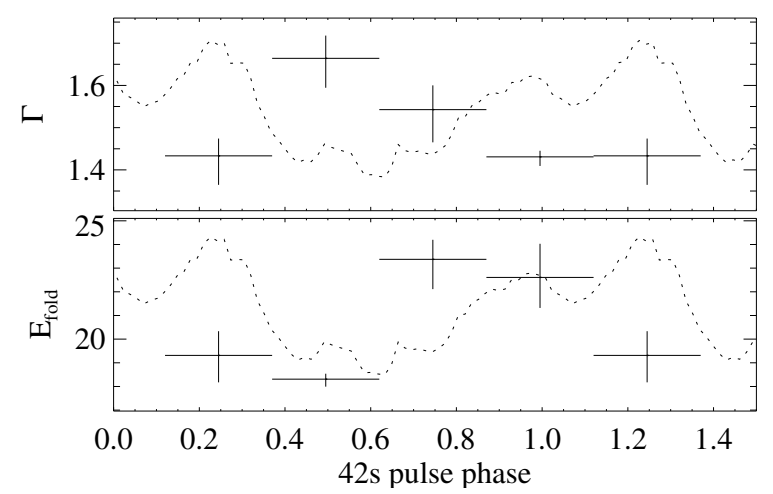

Fig. 10. Best-fit spectral parameters of EXO 2030+375 obtained in Set 3 as a function of pulse phase. The dotted curve shows the averaged ISGRI (20-120 keV) pulse profile. Vertical error bars correspond to $90 \%$-uncertainties.

\section{Interpretation and discussion}

\subsection{Pulse-period derivatives}

The values of pulse-period derivatives measured close to the maximum and at the end of the outburst (Table 2) basically confirm the statement in Klochkov et al. (2007) that the spin-up rate of the pulsar is proportional to its X-ray flux as expected in the simple accretion torque theory (e.g. Pringle \& Rees 1972). The observed dependence is similar to what was measured by EXOSAT during the 1985 giant outburst of EXO 2030+375 (Parmar et al. 1989b). However, with only a few points, it is not possible to explore the correlation using more complicated accretion torque models.

\subsection{Variation in the spectral continuum with pulse phase}

The INTEGRAL observations have shown that the spectral continuum of EXO $2030+375$ is strongly pulse-phase dependent. Observations of the source close to the maximum of the outburst allowed us to perform a detailed study of this dependence. A harder main peak and softer interpulse region observed in the source is a common property of accreting pulsars (see e.g. Tsygankov et al. 2007, and references therein). It is usually explained by assuming that, during the peak, we mostly see the comptonized photons coming from a hot region close to the footstep of the accretion column while in the interpulse softer radiation scattered by the upper parts of the column is observed (Basko \& Sunyaev 1976). A closer look at Fig. 2, however, reveals a more complicated picture. Both the main peak and its left flank increase in relative amplitude with energy, leading to a slight shift of the profile maximum towards an earlier pulse phase. This shows that the spectral continuum changes asymmetrically with respect to the main peak. The variation in spectral parameters with pulse phase (Figs. 5 and 6) demonstrates the corresponding complex pulse-phase dependence of the spectrum. The photon index $\Gamma$ reaches a minimum (the hardest spectrum) in the middle of the main peak (top panel in Figs. 5 and 6), while the maximum of the exponential folding energy $E_{\text {fold }}$ is shifted with respect to the peak towards earlier pulse phases. This contrasts to e.g. Her X-1, where both the maximum of $E_{\text {fold }}$ and the minimum of $\Gamma$ are coincident with the main pulse (Klochkov et al. 2008). 
A possible qualitative explanation for the observed asymmetry of the spectral variation with pulse phase in EXO $2030+375$ can be the following. Since the luminosity of the source close to the maximum of the outburst, $\sim 10^{38} \mathrm{erg} \mathrm{s}^{-1}$, is well above the local Eddington limit (so-called critical luminosity, $L_{\mathrm{c}} \sim$ $10^{37} \mathrm{erg} \mathrm{s}^{-1}$, Basko \& Sunyaev 1976; Staubert et al. 2007), it is expected that accreted matter is decelerated by radiation pressure leading to the formation of an optically thick accretion column. The bulk of the emission in this case is expected to occur as a fan beam (see also the modeling of the source's pulse profiles performed by Parmar et al. 1989a). Therefore, during the maximum of the profile (main peak) the angle between the column axis and the observer's line of sight has the highest value (the column is seen from the side). The observer is looking almost along the beam, seeing the photons coming from a Compton scattering region with large optical depth. This leads to the observed hard power law during the peak. Before the peak, when $E_{\text {fold }}$ reaches a maximum, the direction of the line of sight might be closer to that of the column axis and, thus, of the magnetic field lines. Due to the dependence of the scattering cross-section on the angle between the photon direction and the magnetic field lines (Harding \& Daugherty 1991), one expects that the photons, whose direction in this case is closer to that of the field lines, have experienced less scatterings and, therefore, originated deeper inside the accretion column where the temperature is higher. The X-ray spectrum of these photons is expected to have larger $E_{\text {fold }}$ reflecting higher electron temperature but a softer power-law index due to lower Compton scattering optical depth (see e.g. Rybicki \& Lightman 1979), as observed. This explanation, however, requires that in the latter case the angle between the observer's line of sight and the column axis was not too small. Otherwise, depending on the geometry of the accretion flow, the column density along the line of sight will be very high, resulting in a higher optical depth.

The changing of the pulse profile towards the end of the outburst seems to confirm this picture. In the pulse profiles corresponding to the outburst's decay (Fig. 8), one can see a new peak that appears at the phase interval preceeding the main pulse, i.e. where, according to our view, the observer's line of sight is closest to the magnetic field lines. At this pulse phase one expects to see a pencil beam if the luminosity decreases (Reig \& Coe 1998; Parmar et al. 1989a). The observed peak, therefore, may correspond to the pencil beam component of the emission diagram, whose intensity is comaprable to that of the fan beam component at lower luminosity. Thus, both the pulse phase variation of the spectral continuum during the maximum of the outburst and the evolution of the pulse profile with luminosity fit the described picture where the emission diagram changes from a fan beam geometry close to the maximum of the outburst to a combination of a fan and a pencil beam at the end of the decay phase.

The behavior of the spectral parameters with pulse phase in Set 3 is less clear due to lower statistics (only four phase bins are used). As in the maximum of the outburst, during the main peak of the profile, the spectrum is characterized by a hard $\Gamma$ and a relatively low $E_{\text {fold }}$ (Fig. 10). During the peak around phase 0.0 (pencil beam), $E_{\text {fold }}$ is higher. As before, this can be explained by photons from the pencil beam moving along the magnetic field lines originating deeper in the accretion column where the electron temperature is higher. On the other hand, the emission region is believed to move closer to the star surface as the luminosity decreases (Basko \& Sunyaev 1976). This means that the column density of gas above the emission region will be higher, leading to greater optical depth for Compton scattering. This can explain the hard photon index at this pulse phase.

\section{3. "Bump" versus absorption line at $10 \mathrm{keV}$}

As discussed in Sect. 4.2, to model the spectral continuum of EXO $2030+375$ in the maximum of the outburst, one has to modify the standard power law/cutoff model either by a "bump" at $\sim 15 \mathrm{keV}$ or by an absorption line at $\sim 10 \mathrm{keV}$. Both models provide equally good fits of the pulse-phase resolved spectra. If interpreted as a cyclotron absorption feature (Wilson et al. 2008), the absorption line at $10 \mathrm{keV}$ is observed at rather unusual energy that is much smaller than the exponential cutoff energy $E_{\text {cutoff }}$. This contrasts with all other know cyclotron line sources (see e.g. Fig. 9 in Coburn et al. 2002). We point out here that a feature around 8-15 keV (a "wiggle" or a "bump") is observed in many accreting pulsars (e.g., 4U 1907, Her X-1, Coburn et al. 2002), including those that do not otherwise exhibit a cyclotron line (e.g. GS 1843+00, Coburn 2001). Thus, the interpretation of the absorption line at $10 \mathrm{keV}$ in EXO $2030+375$ as the cyclotron resonant scattering feature might be hasty. On the other hand, the nature of the feature in this and other sources is still unclear. Most probably it arises from modeling the spectral continuum with a simple empirical function. For a proper modeling of the spectrum in the considered energy range (including the feature) one would need a proper theoretical model accounting for all relevant processes at the site of the X-ray emission.

\subsection{Evidence of a cyclotron line at $\sim 63 \mathrm{keV}$}

The X-ray spectrum of EXO 2030+375 taken close to the maximum of the outburst in the narrow pulse-phase interval (0.060.12 ) preceeding the main peak shows evidence of an absorption line around $\sim 63 \mathrm{keV}$ (Fig. 7). If interpreted as a fundamental cyclotron line, the corresponding magnetic field strength is $B \simeq 5 \times 10^{12} \mathrm{G}\left(E_{\mathrm{cyc}} \sim 11.6 \times\left(B / 10^{12} \mathrm{G}\right) \mathrm{keV}\right)$, which is one of the highest values among accreting pulsars. However, evidence of a cyclotron line at $\sim 36 \mathrm{keV}$ has previously been reported for EXO $2030+375$ by Reig \& Coe (1999) during a normal outburst, so the line at $\sim 63 \mathrm{keV}$ might well be the first harmonic rather than the fundamental line. It is known that the relative strength of the fundamental line and harmonics might vary significantly, sometimes making the fundamental line more difficult to detect than the harmonic (a good example is A $0535+26$, see e.g. Kendziorra et al. 1994).

\section{Summary and conclusions}

We used INTEGRAL observations to study the pulse-phase dependence of the broad band X-ray spectrum of EXO 2030+375 close to the maximum and during the decay of its 2006 giant outburst. This is the first pulse-phase resolved spectral study of the source. In all observations, significant pulse phase variability of the X-ray continuum was observed.

Alternative spectral continuum models are discussed. We argue that the interpretation of the feature at $\sim 10 \mathrm{keV}$ as a cyclotron absorption line proposed previously is questionable.

Pulse-phase dependencies of the continuum parameters close to the maximum of the outburst, as well as the evolution of the pulse profiles from the maximum to the end of the outburst, is qualitatively consistent with the picture where the pulsar's emission diagram changes from the fan-beam configuration close to the maximum of the outburst to a combination of pencil and fan beams (whose amplitudes are comparable) at the end of the decay phase.

Evidence of an absorption line at $\sim 63 \mathrm{keV}$ is found during the maximum of the outburst at a narrow phase interval 
preceeding the main peak of the pulse profile. This feature can be interpreted as the first harmonic of the previously reported cyclotron line at $\sim 36 \mathrm{keV}$.

Acknowledgements. This research is based on observations with INTEGRAL, an ESA project with the instruments and science data center funded by ESA member states (especially the PI countries: Denmark, France, Germany, Italy, Switzerland, Spain), Czech Republic and Poland, and with the participation of Russia and the USA. The work was supported by the DLR grant BA5027. We also thank ISSI (Bern, Switzerland) for its hospitality during the team meetings for our collaboration. D.K. thanks Valery Suleimanov (IAAT, Tübingen) for useful discussions.

\section{References}

Arnaud, K. A. 1996, in Astronomical Data Analysis Software and Systems V, ed. G. H. Jacoby \& J. Barnes, ASP Conf. Ser., 101, 17

Bałucińska-Church, M., \& McCammon, D. 1992, ApJ, 400, 699

Basko, M. M., \& Sunyaev, R. A. 1976, MNRAS, 175, 395

Coburn, W. 2001, Ph.D. Thesis, University of California, San Diego

Coburn, W., Heindl, W. A., Rothschild, R. E., et al. 2002, ApJ, 580, 394

Coe, M. J., Payne, B. J., Longmore, A., \& Hanson, C. G. 1988, MNRAS, 232, 865

Corbet, R. H. D., \& Levine, A. M. 2006, The Astronomer's Telegram, 843

Courvoisier, T. J.-L., Walter, R., Beckmann, V., et al. 2003, A\&A, 411, L53

Deeter, J. E., Pravdo, S. H., \& Boynton, P. E. 1981, ApJ, 247, 1003

Ferrigno, C., Segreto, A., Santangelo, A., et al. 2007, A\&A, 462, 995

Hanuschik, R. W. 1996, A\&A, 308, 170

Harding, A. K., \& Daugherty, J. K. 1991, ApJ, 374, 687
Janot-Pacheco, E., Motch, C., \& Pakull, M. W. 1988, A\&A, 202, 81 Kendziorra, E., Kretschmar, P., Pan, H. C., et al. 1994, A\&A, 291, L31 Klochkov, D., Horns, D., Santangelo, A., et al. 2007, A\&A, 464, L45 Klochkov, D., Staubert, R., Postnov, K., et al. 2008, A\&A, 482, 907

Lund, N., Budtz-Jørgensen, C., Westergaard, N. J., et al. 2003, A\&A, 411, L231

Makishima, K., Mihara, T., Nagase, F., \& Tanaka, Y. 1999, ApJ, 525, 978

Mineo, T., Ferrigno, C., Foschini, L., et al. 2006, A\&A, 450, 617

Motch, C., \& Janot-Pacheco, E. 1987, A\&A, 182, L55

Parmar, A. N., White, N. E., \& Stella, L. 1989a, ApJ, 338, 373

Parmar, A. N., White, N. E., Stella, L., Izzo, C., \& Ferri, P. 1989b, ApJ, 338, 359 Pringle, J. E., \& Rees, M. J. 1972, A\&A, 21, 1

Protassov, R., van Dyk, D. A., Connors, A., Kashyap, V. L., \& Siemiginowska, A. 2002, ApJ, 571, 545

Quirrenbach, A., Bjorkman, K. S., Bjorkman, J. E., et al. 1997, ApJ, 479, 477

Reig, P., \& Coe, M. J. 1998, MNRAS, 294, 118

Reig, P., \& Coe, M. J. 1999, MNRAS, 302, 700

Rybicki, G. B., \& Lightman, A. P. 1979, Radiative processes in astrophysics (New York: Wiley-Interscience), 393

Slettebak, A. 1988, PASP, 100, 770

Staubert, R., Shakura, N. I., Postnov, K., et al. 2007, A\&A, 465, L25

Tanaka, Y. 1986, in Radiation Hydrodynamics in Stars and Compact Objects, Proc. IAU Colloq. 89, ed. D. Mihalas \& K.-H. A. Winkler (Berlin: Springer Verlag), Lect. Notes Phys., 255, 198

Tsygankov, S. S., Lutovinov, A. A., Churazov, E. M., \& Sunyaev, R. A. 2007, ArXiv e-prints, 704

Ubertini, P., Lebrun, F., Di Cocco, G., et al. 2003, A\&A, 411, L131

Wilson, C. A., \& Finger, M. H. 2006, The Astronomer's Telegram, 877

Wilson, C. A., Finger, M. H., Coe, M. J., Laycock, S., \& Fabregat, J. 2002, ApJ, 570,287

Wilson, C. A., Fabregat, J., \& Coburn, W. 2005, ApJ, 620, L99

Wilson, C. A., Finger, M. H., \& Camero-Arranz, A. 2008, ApJ, 678, 1263

Winkler, C., Courvoisier, T. J.-L., Di Cocco, G., et al. 2003, A\&A, 411, L1 